\title{
Current Stage in the Development of Klebsiella pneumoniae Vaccines
}

Lucas Assoni · Raquel Girardello · Thiago Rojas Converso •

Michelle Darrieux

Received: May 17, 2021 / Accepted: August 24, 2021 / Published online: September 2, 2021

(c) The Author(s) 2021

\section{ABSTRACT}

Klebsiella pneumoniae is a bacterium capable of colonizing mucous membranes, causing serious infections. Widespread antibiotic resistance in K. pneumoniae-either through intrinsic mechanisms or via acquisition from different species, especially in hospital environments-limits the therapeutic options against this pathogen, further aggravating the disease burden. To date, there are no vaccines available against $K$. pneumoniae infection. Although formulations based on capsular polysaccharides have been proposed, the high variability in capsular serotypes limits vaccine coverage. Recombinant vaccines based on surface exposed bacterial antigens are a promising alternative owing to their conservation among different serotypes and accessibility to the immune system. Many vaccine candidates have been proposed, some of which have reached clinical trials. The present review summarizes the current status of K. pneumoniae vaccine development. Different strategies including whole cell vaccines, outer membrane vesicles (OMVs), ribosome, polysaccharide, lipopolysaccharide (LPS), and protein-based

L. Assoni · R. Girardello · T. R. Converso ·

M. Darrieux $(\square)$

Laboratório de Biologia Molecular de

Microrganismos, Universidade São Francisco,

Bragança Paulista, Brazil

e-mail: sampaiomichelle@uol.com.br formulations are discussed. The contribution of antibody and cell-mediated responses is also presented. In summary, K. pneumoniae vaccines are feasible and a promising strategy to prevent infections and to reduce the antimicrobial resistance burden worldwide.

Keywords: Klebsiella pneumoniae; Vaccines; Vaccination coverage; Enterobacteriaceae

\section{Key Summary Points}

Klebsiella pneumoniae infections represent a major public health threat.

Different vaccine strategies against K. pneumoniae have been investigated and some have reached clinical trials.

The complexity of K. pneumoniae infections suggests multicomponent vaccines as a promising approach to provide long-term immunity with wide coverage.

New technologies are an interesting strategy to refine selection of novel vaccine candidates. 


\section{INTRODUCTION}

Klebsiella pneumoniae is a Gram-negative, encapsulated bacterium frequently found in hospital environments; it is primarily considered an opportunistic pathogen that colonizes human mucous membranes, including the gastrointestinal tract and the oropharynx. From these sites, K. pneumoniae can invade other tissues and cause pneumonia, sepsis, meningitis, liver abscesses, urinary infections, among many other diseases of high significance [1, 2]. $K$. pneumoniae can also cause infections in the community; in particular, the emergence of hypervirulent multidrug-resistant strains (MDR) in the community, such as extended spectrum beta-lactamase (ESBL)-producing and K. pneumoniae carbapenemase (KPC), is cause of great concern worldwide [3-7]. These strains display augmented capsule and siderophore production, as well as increased resistance to antimicrobials. Hypervirulent strains are more prevalent in the community and affect individuals of all age groups, causing diseases in sites where the presence of K. pneumoniae is uncommon, such as endophthalmitis, meningitis, brain, epidural, and splenic abscess and necrotizing fasciitis, in addition to increased risk of other more common infections such as liver abscesses [8]. Of particular importance is the emergence of neonatal infections caused by K pneumoniae, which are associated with high mortality in developing countries around the world [9-11]. According to the Burden of Antibiotic Resistance in Neonates from Developing Societies (BARNARDS) network, K. pneumoniae is the main cause of neonatal sepsis in low- and middle-income countries, accounting for $10 \%$ of total sepsis deaths [12].

Hyperproduction of polysaccharide capsule is the main virulence mechanism reported in K. pneumoniae, contributing to immune evasion and antimicrobial resistance [13]. Capsule production is strictly associated with communityacquired pneumonia and community-acquired urinary infections by K. pneumoniae $[14,15]$. In addition, biofilm formation is an important virulence trait in K. pneumoniae; it can occur in both biotic and abiotic surfaces, as well as within host cells, generating intracellular bacterial communities (IBCs). Biofilms display increased resistance to antibiotics and host immune defenses [16], and promote a favorable environment for horizontal gene transmission [17].

High antibiotic resistance is a hallmark in K.pneumoniae infections. In addition to intrinsic resistance to antibiotics, K. pneumoniae exhibits high levels of horizontal resistance transmission, mainly by conjugative plasmids, which allow the spread of resistance to other microorganisms, of clinical importance such as Acinetobacter baumannii, Pseudomonas aeruginosa, Enterobacter cloacae and Escherichia coli (members of the ESKAPE group) $[18,19]$. Plasmid-associated carbapenemases, including KPC, NDM, IMP, VIM, and OXA-48 enzymes, are disseminated worldwide and cause high rates of morbidity and mortality, varying from $32 \%$ to $65 \%$ [20, 21]. KPC-producing K.pneumoniae is one of the first pathogens isolated from bloodstream infection in patients hospitalized in intensive care units (ICU), a long hospital stay associated with immunocompromised patients being the main risk factor to acquire this pathogen [10].

The combination of increased and widespread antibiotic resistance and the emergence of hypervirulent strains in community-acquired infections place K. pneumoniae as a pathogen of critical risk. According to a global report by the World Health Organization (WHO) [22], the antimicrobial resistance of $K$. pneumoniae in severe healthcare-associated infections is around 50\% worldwide. Despite the recent approval of new antimicrobial options to treat KPC-producing K.pneumoniae-especially ceftazidime-avibactam, a cephalosporin drug associated with a new beta-lactamase inhibitor avibactam-resistance associated with KPC-3 and porin mutations or multiple carbapenemases production has been reported [23, 24].

Thus, there is an urgent need for effective strategies to prevent $K$. pneumoniae infections. Such formulations could prevent both nosocomial and community-acquired infections, especially those associated with hypervirulent strains. K. pneumoniae vaccines could target 
those at increased risk, including hospitalized patients, immunocompromised individuals, and newborns (either directly or through maternal immunization). The next sections summarize the current knowledge on K. pneumoniae vaccines, grouped according to the antigen used in the formulations. An overview of the vaccine strategies under development against K. pneumoniae is presented in Fig. 1.

\section{METHODS}

In the current work, a literature review on $K$. pneumoniae vaccine candidates was conducted through data collected from the NCBI database, using the PubMed search engine (pubmed.ncbi.nlm.nih.gov/), with terms related to the topic, in addition to each of the antigens evaluated and presented in this review. Clinical trial databases were used to search for studies with $K$. pneumoniae in progress; these included the US National Institutes of Health (NIH) (clinicaltrials.gov) and European Clinical Trial Register (EudraCT) (clinicaltrialsregister.eu) along with the ISRCTN (isrctn.com) and

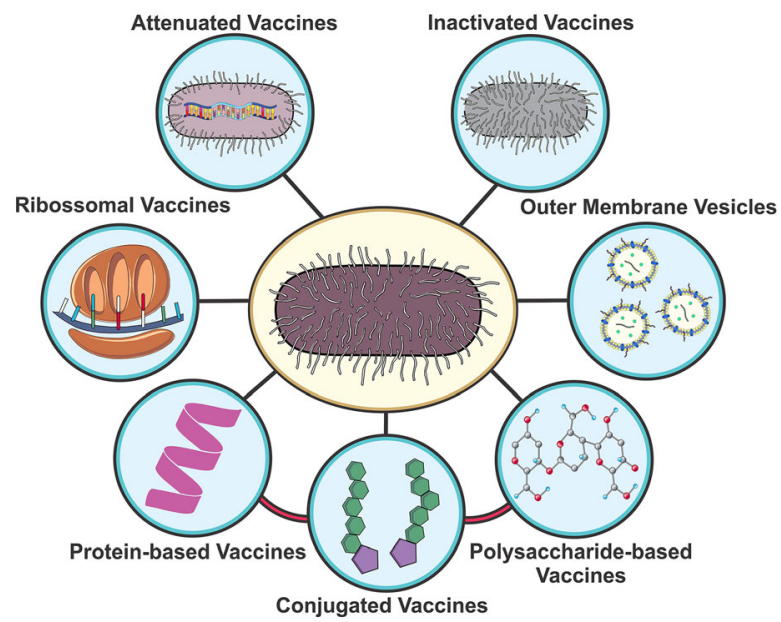

Fig. 1 Vaccine strategies tested against Klebsiella pneumoniae: live attenuated vaccines based on genetically modified bacteria; inactivated whole cell vaccines; outer membrane vesicles containing numerous virulence factors; polysaccharide and lipopolysaccharide (LPS)-based vaccines; protein-based vaccine (recombinant or purified from bacterial extracts); conjugate vaccines including PS-protein or LPS-protein fusions; ribosomal vaccines
Global Clinical Trials Data (globalclinicaltrialsdata.com) search engines.

No exclusion date was determined for the selection of the publications because of the limited sources of information related to the advances in the development of K. pneumoniae vaccines. The search of the bibliography was conducted between September of 2020 and May of 2021.

This article is based on previously conducted studies and does not contain any new studies with human participants or animals performed by any of the authors.

\section{WHOLE CELL VACCINES/CELL LYSATES}

Whole cell K. pneumoniae vaccines have been investigated alone or in combination with other enterobacteria. These formulations include live attenuated bacteria, inactivated whole cells, and cell extracts.

Mutant K. pneumoniae strains lacking the housekeeping gene ton $B$ were protective against challenge when administered intraperitoneally [25]. This mutant displayed reduced ability to acquire iron from the extracellular environment; however, it was still able to secrete siderophores, which played a role in lung pathology and bacteria dissemination in a mouse pneumonia model, as a result of the release of proinflammatory cytokines interleukin-6 (IL-6), CXCL1, CXCL2, IL-1 $\beta$, and macrophage inflammatory protein 3 alpha (MIP-3 $\alpha$ ) [26].

The mouse pneumonia model was also employed to evaluate protection mediated by an inactivated K. pneumoniae vaccine. Intranasal vaccination induced the production of IgG and IgM and protected against nasal challenge. Interestingly, protection did not seem to be mediated exclusively by antibodies against the capsule; a serotype-independent, unidentified antigen was also protective in this model. IgG was also able to induce passive protection against intraperitoneal challenge [27].

Mixed bacterial vaccines (MBVs) are formulations containing heat-killed pathogens. One of these formulations includes Haemophilus influenzae, Neisseria catarrhalis, Streptococcus 
pyogenes, K. pneumoniae, Staphylococcus aureus, and Streptococcus pneumoniae strains. In an infectious asthma study in children, immunization with the MBV reduced the frequency and severity of infections, with its effect lasting for years. The findings were attributed to the hyposensitization generated by the low bacterial dosage, which was best tolerated by the participants [28]. However, in a clinical trial with 95 institutionalized male individuals, the formulation failed to induce protection against respiratory infections [29].

A double-blind clinical trial using tablets containing either bacterial lysates of $S$. pneumoniae, H. influenzae, K. pneumoniae, Klebsiella ozaenae, S. aureus, S. pyogenes, Streptococcus viridans, and Neisseria catarrhalis (OM-85 BV) or ribosomal fractions derived from $S$. pneumoniae, S. pyogenes, H. influenzae, and K.pneumoniae (D53) showed an induction of anti-K. pneumoniae antibody-producing cells in the tonsils of children between 3 and 16 years old. The ribosomal formulation showed superior results when compared to the whole cell vaccine [30]; however, the findings were later contested [31]. Ribosomal-based vaccines will be discussed in a separate section.

Another MBV is Dentavax, a formulation composed of inactivated cells from K. pneumoniae, $S$. pyogenes, $S$. aureus, Candida albicans, and Lactobacillus acidophilus, developed to prevent colonization and inflammation of the oral mucosa. In the study, 12 volunteers were given tablets containing the mixture, and systemic and mucosal responses were observed. An increase in antibody production was detected in serum (IgG) and saliva (IgA) from immunized subjects, as well as tumor necrosis factor alpha $(\mathrm{TNF} \alpha)$ production by peripheral blood lymphocytes [32]. Similarly, Respivax was designed to prevent respiratory infections and includes inactivated S. pneumoniae, N. catarrhalis, S. pyogenes, H. influenzae type B, S. aureus, and K. pneumoniae strains. The 25 participants enrolled in the clinical trial had recurrent infections and demonstrated reduced expression of immune cell receptors when compared to healthy individuals. Immunization with the MBV showed a protective immunomodulation, inducing expression of TLR2, CD14, CD86, and
HLA-DR in monocytes and polymorphonuclear leukocytes (PMNs). Interferon- $\gamma$ (IFN $\gamma$ ) and $\mathrm{TNF} \alpha$ expression profile was also restored to levels close to those showed in healthy individuals. No major adverse reactions were reported up to 6 months following the study [33].

Vaccines based on inactivated enterobacterial strains were able to protect against recurrent urinary tract infections (UTIs). Immunization with Uromune ${ }^{\circledR}$, a sublingual spray containing inactivated K. pneumoniae, E.coli, Proteus vulgaris, and Enterococcus faecalis, provided protection against recurrent UTI in women in a phase II study. Adverse reactions related to the formulation were only reported with one participant, while small potential adverse reactions occurred in seven participants [34]. A similar study in Spain, with 784 patients, mostly women, reported comparable results in the reduction of recurrent UTIs in the individuals, with minimum side effects reported [35]. The specific immune responses induced by this formulation were not investigated in those studies.

Another multivalent formulation to prevent UTIs, Urovac ${ }^{\circledR}$, comprises E. faecalis, K. pneumoniae, Morganella morganii, Proteus mirabilis, and six more E. coli strains administered locally, via vaginal suppositories. In phase II, randomized, blinded, and placebo-controlled trials, the period between reinfections within the group immunized with boosters was longer when compared to the placebo and the vaccine plus placebo dose group during the 6 months study period. The strongest correlation was found in sexually active volunteers with recurrent urinary infections. The vaccines were well tolerated, and no severe adverse reactions were reported [36-38]. Oral administration of Urostim ${ }^{\circledR}$-another formulation composed of E. coli, K. pneumoniae, P. mirabilis, and E. faecalis-reduced reinfections with these bacteria in 320 participants with recurrent or chronic UTIs, pyelonephritis, or prostatitis, an effect that correlated with increased secretory $\operatorname{IgA}$ and phagocytosis [39]. Other MBVs include Ismigen $^{\circledR}$ as prophylaxis or as a coadjuvant in the treatment of chronic obstructive pulmonary disease [40-44], and Lantigen B to prevent respiratory and ear infections and eczemas. 
Despite the improved health of the patients, no specific immunological parameters were evaluated [45-47].

Taken together, whole cell vaccines have shown to be a promising approach to prevent respiratory and urinary infections by K. pneumoniae; however, many of these formulations display toxicity, which limits their widespread use. The length of the acquired protection and the immunological mechanisms induced by these vaccines have not been fully demonstrated.

\section{Subunit Klebsiella pneumoniae Vaccines}

\section{Capsular Polysaccharides}

Recently, many reviews have explored the possibility of a capsular polysaccharide-based vaccine to prevent K.pneumoniae infections [48-51]. The high immunogenicity and surface exposure of capsules make them an interesting vaccination strategy; however, the structural variability and variations in geographic distribution of serotypes limit the potential coverage of these formulations [52-54]. Currently, 77 capsular serotypes (referred to as K polysaccharides) have been identified in K. pneumoniae, with many other serotypes deriving from rearrangements in the cps and $k$ loci (known as the KL series) [51].

Passive immunization using antibodies raised against the polysaccharide capsule (CPS) protected mice against sepsis and pneumonia. Similarly, formulations containing membrane polysaccharides (PS) were able to induce protection in animal models of infection [48]. A bioconjugation approach based on glycoengineered E.coli expressing K. pneumoniae $\mathrm{K} 1$ and $\mathrm{K} 2$ antigens under regulation of $r m p A$ (a regulator of mucoid phenotype A) led to production of IgG against both glycans in mice and protected against lethal challenge with $\mathrm{K} 1$ and $\mathrm{K} 2$ strains [55].

Phase I and II clinical trials were performed using K. pneumoniae capsular polysaccharides, either alone or co-administered with a $P$. aeruginosa conjugate formulation. CPS from a K1 strain was able to induce IgM and IgG production in healthy volunteers between 16 and
59 years old that lasted throughout the study3 months for IgG and 2 months for IgM. Most of the adverse reactions were local and only 13 of the 72 participants reported broader symptoms, namely fever, headaches, and malaise. Passive immunization with anti-CPS IgG from sera of the volunteers was also able to partially protect mice against sepsis in a burn wound model [56]. A 24-valent CPS formulation from K.pneumoniae and Klebsiella oxytoca was administered in addition to an 8-valent $P$. aeruginosa $\mathrm{O}$ antigen conjugated with Pseudomonas toxin A as a carrier protein. The IgG response against the different serotypes persisted for 18 months after the first immunization [57]. The same formulations were administered alone or in addition to tetanus toxoid, in a clinical study of patients with acute trauma. Although the number of volunteers was small (10 patients), the vaccines were well accepted, with minimum side effects, and the overall vaccine response was encouraging: all but one PS (K35) induced a fourfold increase in antibody levels in at least $70 \%$ of the patients, while seven patients reacted against 22 of 24 of the serotypes [58].

A recent study investigating synthetic K2 capsular polysaccharides conjugated with diphtheria toxin (DT) demonstrated production of specific anti-capsular antibodies in mice which displayed in vitro bactericidal activity through complement system activation [59]. However, in vivo protection was not evaluated in that study.

Finally, Malachowa et al. recently developed a pneumonia model to evaluate the protective potential of vaccines based on purified capsule polysaccharide type 2 (CPS2) in cynomolgus macaques. The vaccinated animals developed anti-capsular antibodies which enhanced in vitro serum bactericidal activity and opsonophagocytosis by neutrophils. They also reported an increase in chemokines and inflammatory cytokines such as IL-17A, which correlated with reduced bacterial loads in the lungs [60].

Polysaccharide-based vaccines have been successfully used to prevent disease by both Gram-negative and Gram-positive bacteria for decades; their general high immunogenicity 
and external cellular localization make polysaccharides excellent immune targets. Nevertheless, the high variability in capsule polysaccharide composition greatly impacts vaccine coverage, while their inability to promote $\mathrm{T}$ cell-dependent immune responses hinders vaccine efficacy, especially in young children, who are not able to respond to T cellindependent antigens. This latter issue may be overcome by polysaccharide conjugation to protein carriers, although this will greatly improve vaccine cost. This approach has been successfully used in the development of vaccines against $S$. pneumoniae-a major human pathogen with over 100 capsular serotypes [61]. The great impact of pneumococcal conjugate vaccines-composed of 10 or 13 most prevalent capsule polysaccharides fused to protein carriers-in reducing disease burden across the world [62] suggests that careful selection of the most clinically relevant serotypes is a promising strategy to prevent disease by encapsulated bacteria such as K. pneumoniae.

\section{Lipopolysaccharides}

The $\mathrm{O}$ antigen is an important virulence factor in K.pneumoniae, directly related to survival success after invasion of the host. There are 11 different $\mathrm{O}$ antigens found in K. pneumoniae, a reduced number when compared to the variety of polysaccharides that compose the capsule. The $\mathrm{O} 1, \mathrm{O} 2, \mathrm{O} 3$, and $\mathrm{O} 5$ antigens are more commonly present in the clinically relevant strains, with $98 \%$ of the isolates being of serotypes O1 to O4 [51]. Since many K. pneumoniae serotypes share a similar LPS backbone structure, formulations targeting such structures can be promising strategies [63].

Many different formulations based on LPS have been evaluated in different models of K. pneumoniae infection. Extracted LPS was evaluated against lobar pneumonia in mice and was able to reduce lung burden following challenge; however, it was also pyrogenic in a rabbit temperature assay. Interestingly, the reduction of carboxyl groups of LPS reduced the pyrogenicity of the formulation and the conjugation of bovine serum albumin (BSA) with either lipid A or the extracted LPS inhibiting the increase in the rabbit's temperature while retaining the protection ability at a 100 -fold dose reduction. All formulations induced in vitro bacterial killing by macrophages [1]. Another pneumonia mouse model using LPS showed that intranasal and intramuscular administration of the vaccine reduced the bacterial burden in the lungs; however, lung tissue damage was reported [2]. The use of microparticles of sodium alginate containing LPS had similar effects reducing the lung bacterial burden; however, the tissue damage was higher when compared to the free LPS administered intranasally, intramuscularly, or intratracheally [3]

Rat pneumonia models were also used to assess the protective potential of LPS associated with liposomes. This strategy was able to confer protection, reducing the lung bacterial load, while reducing the toxicity of LPS; whereas the LPS alone and combined with liposomes were pyrogenic in a rabbit assay, the incorporation of LPS in the liposome nullified the pyrogenic activity of the formulation [4].

Detoxified LPS from E. coli complexed with a Neisseria meningitidis outer membrane protein (OMP) increased survival against K. pneumoniae and $P$. aeruginosa challenge and prevented sepsis in neutropenic rats [64]. The formulation also induced higher IgG levels when compared to extracted and detoxified Brucella abortus LPS [5].

Similarly, a liposome formulation composed of E.coli, P. aeruginosa, and Bacteroides fragilis complete-core LPS and lipid A protected mice against lethal challenge. Sera from rabbits previously immunized with the liposomal formulation reacted against multiple K. pneumoniae strains with different $\mathrm{O}$ antigen serotypes [65]. Anti-E. coli mAbs demonstrated comparable results, cross-reacting with $K$. pneumoniae lipid A and Serratia marcescens LPS [66]. Purified antiLPS or monoclonal antibodies against LPS antigenic units granted immunity against systemic and pulmonary infection in mice, reducing the bacterial burden in liver, spleen and lungs. Cross-reaction of antibodies against LPS was observed among strains containing different types of $\mathrm{O}$ antigen [67-70].

Intramuscular or intraurethral administration of K. pneumoniae LPS was also protective in a UTI mouse model. Both immunization routes 
led to a reduction in bacteria colonizing the kidneys [71].

Besides being used as a vaccine antigen, LPS has been evaluated as an adjuvant in conjugation with iron-regulated, cell surface proteins. The conjugated vaccine reduced bacterial burden in a pneumonia rat model and increased bacterial phagocytosis [72]. Another conjugate vaccine included K.pneumoniae $\mathrm{O} 1$ coupled with $P$.aeruginosa flagellin subunits FlaA and FlaB. The glycoconjugate induced high IgG levels against $\mathrm{O}$ antigen, and sera from immunized mice was able to reduce the bacterial burden in blood, liver, and spleen and mortality in mice. In rabbits, the conjugation of FlaA or FlaB to O1, O2, O3, or O5 increased the antibody response against $\mathrm{O}$ antigen when compared to the group injected with the coadministered antigens [73].

Finally, a formulation including antigens $\mathrm{O} 1, \mathrm{O} 2, \mathrm{O} 3$, and $\mathrm{O} 5$ coupled with the type III fimbriae adhesin MrkA composing a multipleantigen presenting system (MAPS) is under development by Affinivax [74].

Lipopolysaccharides are highly immunogenic molecules and important virulence factors for K. pneumoniae. They are surface exposed and considerably less variable when compared to polysaccharides. Vaccine formulations utilizing LPS or LPS fragments have been successfully tested in different models of infection. However, the high toxicity of LPS is the main limiting factor related to this type of vaccine. Detoxification protocols have been used to enhance vaccine safety, but a delicate balance between immunogenicity and toxicity must be considered. Furthermore, LPS, such as polysaccharides, are $\mathrm{T}$ cell-independent antigens which are not suitable for all age groups. This can be overcome by conjugation to protein carriers, which have been shown to promote strong, lasting responses in children as previously described.

\section{Outer Membrane Vesicles}

Like many Gram-negative bacteria, K. pneumoniae produce outer membrane vesicles (OMVs), which constitute an important virulence factor for this bacterium. OMVs comprise a lipid bilayer vesicle containing LPS, lipids, cell wall structures, nucleic acids and proteins, such as OMPs, cytoplasmatic proteins, and toxins $[75,76]$. OMVs are also related to immune system evasion and extracellular transport of biomolecules of interest [77]. Vaccine formulations containing OMVs and/or OMPs have been tested in different infection models.

K. pneumoniae OMVs were able to induce cellular immune responses in bronchial epithelial cells, with increased production of IL-1 $\beta$, IL8, IL-6, and TNF $\alpha$. [76]. Similar results were found for laryngeal epithelial cells, where OMVs induced IL-1 $\beta$ and IL-8. In an immunocompromised mouse model, the intratracheal inoculation of OMVs promoted pulmonary pathology, suggesting a strong and potentially harmful inflammatory response [75]. One of the reasons behind the immunogenicity of OMVs is the release of OMPs (OmpA and OmpC) inside the vesicles, which contribute to the inflammatory response [76]. Cytokine production by mouse macrophages was also enhanced in the presence of OMVs containing secreted OmpK35 and OmpK36, with expression of TNF $\alpha$, macrophage inflammatory protein 2 , and granulocyte-macrophage colony-stimulating factor [78]

In vitro assays demonstrated the production of IL- 6 and TNF $\alpha$, along with overexpression of MHC II and CD86 in dendritic cells upon stimulation with OMVs [79]. The same study showed that the in vivo response to OMVs included a mixed Th1, Th2, and Th17 profile. Immunization with OMVs prevented septic death after challenge. Protection was also achieved via passive immunization with sera or splenocytes from immunized mice [79]. The addition of BSA nanoparticles to K. pneumoniae OMVs increased the protective potential against lethal challenge with KPC, which correlated with higher IgG production. Along with the in vivo protection, the combination boosted the recruitment and activation of dendritic cells, with overexpression of MHC II, CD40, CD80, and CD86. In vitro assays showed increased secretion of IL-6, TNF $\alpha$, and IFN $\gamma$ by mouse macrophages after stimulation with the OMVs. IFN $\gamma$ levels were especially higher when compared to the non-BSA-paired OMV group [80]. 
OMVs are a promising approach in vaccine development, since they induce specific adaptive immune responses through the presence of OMPs, and toxins, while displaying intrinsic adjuvant properties. OMVs have been used as platforms for different vaccines, being particularly interesting as mucosal delivery systems [81]. Although OMVs are usually well tolerated and display low toxicity, the pulmonary pathology observed with intratracheal injection of K. pneumoniae OMVs raises safety concerns and suggests further studies are needed to reduce undesirable side effects. Furthermore, the complexity of OMV composition, with the presence of multiple antigens in variable concentrations makes it difficult to ensure consistency in the manufacturing procedures and may affect quality control.

\section{Ribosomal-Based Formulations}

Vaccine formulations based on purified bacterial ribosomes or ribosomal fractions have been investigated for many decades, and in general promoted increased protection when compared to whole cell vaccines [82].

Purified ribosomes, proteins extracted from ribosomes, or extracted ribosomal RNA from K. pneumoniae showed protection in a systemic lethal challenge model when injected subcutaneously [83]. Passive immunization with spleen cells was protective in a lethal challenge. However, hyperimmune serum was not protective in this model, suggesting that the cellular response is the predominant protective mechanism induced by these formulations [84].

An aerosol formulation based on ribosomes of K.pneumoniae, S.pneumoniae, S.pyogenes, $H$. influenzae, in addition to membrane glycoproteins of K. pneumoniae, induced an increase in IgG and IgM levels for at least 255 days after the first dose in the vast majority of the volunteers [85]. A similar formulation, Ribomunyl, was administered orally in patients with recurrent infectious rhinitis. The treated group showed reduced incidence compared to the placebo-treated individuals over a 6-month study period [86]. In a similar, double-blind clinical trial, ribosomal fractions derived from S. pneumoniae, S.pyogenes, H. influenzae, and K. pneumoniae were given to 90 children between 3 and 16 years old, who had undergone tonsillectomy. The tablet formulation named D53 stimulated specific anti-K. pneumoniae antibody-producing cells at a higher level than the bacterial lysate formulation OM-85 used in the same study [30]. As described in the OM-85 section, the results of the study were later questioned [31].

Overall, the protective efficacy of ribosomebased vaccine formulations is controversial, since many include surface protein contaminations, which may be major contributors to the protective responses. The ribosomes may act as adjuvants for these surface protein contaminants or, in some cases, carry mRNA for these proteins which is then translated in the immunized host [82]. Ribosomal fractions also display immunomodulatory effects, with the induction of $\mathrm{T}$ cell responses that contribute to $\mathrm{B}$ cell proliferation and increased antibody production [87].

\section{Protein-Based Formulations}

Outer Membrane Proteins Various formulations based on recombinant OMPs have been evaluated for their protective effect against K. pneumoniae infections. OmpA (also known as $\mathrm{P} 40$ ) has been suggested as a potential carrier in conjugated formulations, with similar efficiency as tetanus toxoid [88]. When administered as a carrier in combination with respiratory syncytial virus subgroup A (RSV-A), it induced IgA, IgG1, and IgG2a production which were protective against challenge [89]. These findings are supported by in vitro assays showing the upregulation in production of $\mathrm{TNF} \alpha$ and nitric oxide in vaccinated mice, as well as increased bacterial attachment and internalization by human macrophages [90]. Similar results were found after conjugation with a peptide derived from RSV attached to G protein and an $H$. influenzae type B PS. Interestingly, immunization with recombinant OmpA induced a mixed Th1/ Th2-type response, including cytokines such as IL-2, IL-5, IL-10, and IFN $\gamma$ [91].

A vaccine including OmpA fused to OmpK36 led to an increase in secretion of IL-2 and IFN $\gamma$ and protected mice from K. pneumoniae challenge. Sera from immunized mice was able to prevent bacterial biofilm formation in vitro. 
Despite the encouraging results, the induced antibodies showed cross-reactivity with OMPs present in other Enterobacteriaceae strains, which could lead to undesirable effects on the host microbiota [92]. OmpK17 and OmpK36 were able to protect against lethal challenge and induced IgG1 and IgG2a; a chimera formulation of both proteins induced a Th2-type response with strong IgG1 production [93].

Similarly to the results using recombinant OMPs, mouse immunization with a DNA vaccine including ompA and $o m p K 36$ was capable of inducing an increase in IgG and interleukins, and conferred protection against lethal challenge [94]. Extracted, LPS-free OMPs from a CPS-deficient strain protected against lethal challenge with a wild-type strain, and a similar effect was observed with passive immunization using OMP-induced antibodies [95].

In silico analyses have also demonstrated that OMPs are promising candidates for a Klebsiella vaccine [96]. A study using reverse vaccinology selected candidates for heterologous expression from K. pneumoniae antigens with predicted $\mathrm{T}$ and $\mathrm{B}$ cell epitopes. The epitopes were fused in a chimera, using cholera toxin subunit B as adjuvant. Among the epitopes, those derived from OmpA and PhoE (an outer membrane porin) were among the most promising candidates; however, to date, the formulation was still not tested in vivo [97]. Proteomic analysis showed that OmpN and CusC are interesting vaccine candidates, since they are highly conserved among different strains and predicted to be antigenic, while displaying no homology to proteins found in members of the human microbiota [98].

OmpA was also proposed as an adjuvant in melanoma vaccines, which have undergone phase I clinical trials [99]. Another study evaluated OmpA as a carrier protein following conjugation to S. pneumoniae CPS 14 and 19. The addition of OmpA to the pneumococcal polysaccharides increased IgM and IgG production, mainly IgG1 and IgG3, against S. pneumoniae CPS 14 and 19 [100]. Passive immunization with anti-OmpA-CPS19 antibodies was partially protective against pneumococcal challenge.
In sum, the literature indicates that recombinant OMPs are promising vaccine candidates against K. pneumoniae, alone or combined with other antigens. Owing to their adjuvant effects, these proteins can also act as immunomodulatory agents in cancer vaccines. However, the cross-reactivity with molecules expressed by other enterobacteria suggest a possible interference with the host microbiota, which could be overcome with bioinformatic tools to select specific immunogenic epitopes that are not shared by commensal bacteria.

Fimbriae Subunits In K.pneumoniae, two main types of fimbriae are found, type I and type III. The fim gene cluster is responsible for type I fimbriae, which are related to adhesion to the epithelium and to mucous membranes [2]. The fimbriae components encoded by the $m r k$ gene cluster are responsible for the type III fimbrial subunits, which are essential for adhesion and biofilm formation on biotic and abiotic surfaces [101, 102]. Other fimbriae clusters have been identified [103], although some of these new clusters are part of the accessory genome, and their distribution is species-dependent [2].

Type I fimbriae extracted from K. pneumoniae and type III fimbriae from K. oxytoca were evaluated as protein carriers in conjugation with E. coli endotoxin. Although the level of TNFa secreted by murine fibroblasts was similar to that induced by endotoxin alone, there was a slight increase in the production of IL- 6 and IFN in the presence of both fimbriae conjugates, in A549 and 7TD1 cells, respectively [104].

In K.pneumoniae, immunization using recombinant MrkD or MrkD-derived peptides was capable of activating $\mathrm{CD} 4^{+} \mathrm{T}$ lymphocytes, with production of IL-4 and IFN $\gamma$ [105]. In another study, immunization with recombinant MrkA or anti-MrkA monoclonal antibodies induced protection in mice against pneumonia. These monoclonal antibodies reduced in vitro biofilm formation and cell adhesion, cross-reacted with strains of different serotypes, and promoted bacterial opsonophagocytosis $[106,107]$.

Immunization using purified type III fimbriae from K. pneumoniae was protective in a 
mouse pneumonia model, using a low dose of bacteria. Protection was correlated with specific IgG production; however, other protection parameters were not evaluated. Passive immunization was also achieved via administration of sera from hyperimmune animals [108].

In E.coli, fimbrial subunits have been extensively investigated as potential vaccine candidates. Immunization using E. coli type I fimbriae subunits/adhesins showed promising results against UTIs in various animal models [109-117]. FimH was also tested as a protein carrier in a chitosan nanoparticle, DNA vaccine against coxsackievirus. The addition of FimH increased the survival of mice and induced the production of fecal sIgA and serum IgG, in addition to stimulating $\mathrm{T}$ cell proliferation in spleen and lymph nodes. Increase in IFN $\gamma$ in splenocytes was also observed, along with higher specificity of cytotoxic T cells. mRNA overexpression of CD80, CD86, and MHC II was reported in dendritic cells [118].

Generally, fimbriae components have been shown to be promising candidates for inclusion in vaccines. Their contribution to important virulence traits, such as biofilm formation and adhesion to host cells, in addition to their surface exposure, reinforces their potential to elicit protective immune responses. However, since most studies were conducted using E. coli, further work is needed to assess the protective role of recombinant fimbriae subunits in different models of K. pneumoniae infection. Owing to the high similarity between these two species of enterobacteria, an approach targeting the highly conserved fimbriae genes of K. pneumoniae could provide interesting results.

Toxins K.pneumoniae (along with other members of the ESKAPE group) produce and secrete toxins and anti-toxins, especially the hypervirulent strains. These biomolecules are related to virulence, including an increased survival in the presence of antimicrobials [119]. One toxin in particular, colibactin, is genotoxic and able to hinder the host cell cycle [120]. Such deleterious activity has been implicated in colorectal cancer development and neonatal systemic infections, namely sepsis and meningitis [121]. The important role of such toxins in disease pathogenesis makes them ideal targets for vaccine development, and many have been investigated in animal models.

Purified heat-stable K. pneumoniae enterotoxin was able to protect against lethal challenge, with similar results to those induced by E. coli enterotoxin in rats [122]. Toxoids from K. pneumoniae derived from endogenous cytotoxin I promoted protection in a systemic model of infection in mice. Among the toxoids, KCT-I and PBE provided protection against K. pneumoniae and K. oxytoca strains. In female rabbits, the immunization induced elevated IgG levels, which were later also found in their offspring's serum. Similarly, passive immunization using colostrum from previously immunized animals was protective to newborn rabbits, after lethal challenge [123], demonstrating a role for the antibody response in this model.

Toxins have been traditionally used as human vaccines. The DTaP vaccine includes tetanus and diphtheria toxoids combined with acellular pertussis, and is administered to children in many countries, with booster doses of DT recommended for adults. Toxins make excellent vaccines owing to their high immunogenicity and pivotal role in disease pathogenesis. The main concern is their intrinsic toxicity, which must be abrogated to allow their safe utilization. Several detoxification protocols, including physical treatments, chemical inactivation, and site-directed mutagenesis, may be employed to produce toxoidsthe non-toxic yet immunogenic form of the toxins.

Other Proteins Siderophores and membrane receptors are interesting vaccine candidates owing to their cellular location-which allows prompt recognition by the host immune system-and contribution to virulence. Therefore, different molecules involved in nutrient/ion transport and environmental sensors have been proposed as vaccine candidates.

Siderophore receptor protein (SRP) was able to protect against bacterial bovine mastitis caused by Klebsiella, as well as other relevant coliforms [124]. FepA, a protein related to siderophore activity, was proposed as a potential candidate after immunoproteomic analysis of 
infected patients [94]. FepB, a enterobactin transporter, was chosen among many conserved proteins as a potential candidate using reverse vaccinology [98]. A conjugated vaccine composed of iron-regulated, cell surface proteins and LPS as an adjuvant induced antibodies with increased phagocytic ability and reduced the bacterial burden in a pneumonia rat model [72].

FyuA is a conserved yersiniabactin receptor of Enterobacteriaceae, directly related to siderophore expression. Immunization with FyuA prevented pneumonia in a mouse model, an effect that correlated with an early increase in IL-17, IL-1 $\beta$, and TNF $\alpha$ in the lungs after the first day of infection, followed by a rapid decrease on the next day. Serum from the immunized group also promoted an increase in bacterial phagocytosis and higher bactericidal activity [125].

Antigenome analyses revealed proteins conserved among different serotypes as potential vaccine candidates, including RecX, $\mathrm{RecO}$, YhiN, and YfhM. DNA vaccines including genes from these antigens were effective in a sepsis model [126]. Colicin I, a bacteriocin receptor, is predicted to be highly immunogenic and suggested as a potential target in vaccine formulations, following an immunoproteomic analysis of a clinical, drug-resistant K. pneumoniae strain; however, the protective potential of the protein was never evaluated [127]. Another proteomicbased analysis suggested the zinc transporter ZnuA, the ribonuclease $\mathrm{H} 1$, the TehB methyltransferase, and two more hypothetical proteins (WP_002918223 and WP_002892366) as candidates for a vaccine formulation, based on conservation among strains and immunogenicity prediction [98].

Recently, another candidate, the predicted adhesin YidR, has been tested in different animal models. The protein was able to protect mice against lethal challenge [128] and improved bovine milk production via protection against Klebsiella and E. coli infections [129]. In E. coli bovine mastitis isolates, the ferric citrate receptor FecA was found to be overexpressed. Serum from rabbits immunized with FecA showed binding to E.coli, and also to K. pneumoniae mastitis isolates in an immunoblot assay [130]. Cow immunization with FecA was able to induce high titers of IgG.
Subsequently, purified IgG showed the ability to prevent iron acquisition from E. coli isolates [131]. The ferric enterobactin receptor FepA exhibited similar iron acquisition blocking to FecA, with synergistic activity when combined [132]. In a cow mastitis model induced via local inoculation of E. coli, though FecA was deemed immunogenic, it had no effect on the clinical manifestations [133].

Surface environmental sensors, proteins involved in nutrient uptake, or molecule secretion systems are interesting vaccine candidates, because the induction of immune responses that limit their functions will affect the pathogen's ability to colonize the host. In addition to the well-studied siderophores and transport systems, bioinformatics has provided many new potential vaccine candidates on the basis of their predicted cell localization and function. Although further studies are needed to confirm which of those molecules provide the best, long-term immunity, data from other pathogens such as N.meningitidis has proven the success of this new technology in vaccine development.

\section{CLOSING REMARKS}

Infections caused by K. pneumoniae represent a major threat to public health worldwide. In the past, K. pneumoniae was mainly considered an opportunistic pathogen; however, the rapid increase in community-acquired Klebsiella infections, including multidrug-resistant strains [3-7], has placed this bacterium amongst the top pathogens. Therefore, the development of vaccines against $K$. pneumoniae has become a priority in many countries [49].

As previously shown for several pathogens, vaccination is an effective way to prevent disease, especially when considering those more susceptible-the elderly, children, and immunocompromised individuals. Several approaches have been explored to prevent K. pneumoniae infections, including whole cells and cell extracts, ribosomal vaccines, and virulence factor including OMVs, capsular polysaccharides, LPS, and proteins (Fig. 1). Some of 
these formulations have been successful and progressed to clinical trials.

While all vaccine approaches have limitations-these being the increased reactogenicity of whole cell vaccines or the lower immunogenicity of subunit formulations-there is increasing evidence that $K$. pneumoniae vaccines can be a reality within the next decades. Particularly, the use of conjugate vaccines including capsular polysaccharides and/or $\mathrm{O}$ antigens fused to protein carriers is an alternative with great potential. The careful selection of the most clinically relevant capsular and O serotypes is key to developing effective vaccines with broad coverage. Also, since essential proteins have a tendency to be conserved within the species, the inclusion of K. pneumoniae surface proteins as carriers could prevent serotype replacement in the long term [134], while increasing the protective efficacy of the formulations.

The development of subunit vaccines has been greatly impacted in recent years by new techniques to identify and validate potential candidates. The antigenome strategy, combining informatic tools with knowledge on disease pathogenesis, has provided a great number of new potential vaccine targets to be explored. These molecules can be tested alone or combined to generate multicomponent vaccines to tackle different disease stages or niches.

In conclusion, there is an urgent need for effective K. pneumoniae vaccines that are able to protect against the increasing numbers of multidrug-resistant and hypervirulent strains found in the community. Given the complexity of K. pneumoniae infections, it is plausible to assume that different formulations will be generated to prevent diverse forms of disease. Multicomponent conjugate vaccines comprise the most promising strategy to provide longterm, broad vaccine coverage. The use of recombinant systems allows safe production of vaccinal antigens with high yields, while the combination of genomic/proteomic analysis and better understanding of disease pathogenesis refines the selection of new candidates.

\section{ACKNOWLEDGEMENTS}

Funding. Lucas Assoni has a scholarship from Coordenação de Aperfeiçoamento de Pessoal de Nível Superior (CAPES) (grant 88887.601281/ 2021-00). No Rapid Service Fee was received by the journal for the publication of this article.

Authorship. All named authors meet the International Committee of Medical Journal Editors (ICMJE) criteria for authorship for this article, take responsibility for the integrity of the work, and have given their approval for this version to be published.

Author Contributions. The authors contributed to the present work as follows. Literature search (LA and MD), Original draft formulation (LA, TRC and MD). Review of the manuscript (RG, TRC and MD). Conceptualization of the figure (LA and MD). Overall supervision of the work (TRC and MD).

Disclosures. Lucas Assoni, Raquel Girardello, Thiago Rojas Converso and Michelle Darrieux have nothing to disclose.

Compliance with Ethics Guidelines. This article is based on previously conducted studies and does not contain any new studies with human participants or animals performed by any of the authors.

Open Access. This article is licensed under a Creative Commons Attribution-NonCommercial 4.0 International License, which permits any non-commercial use, sharing, adaptation, distribution and reproduction in any medium or format, as long as you give appropriate credit to the original author(s) and the source, provide a link to the Creative Commons licence, and indicate if changes were made. The images or other third party material in this article are included in the article's Creative Commons licence, unless indicated otherwise in a credit line to the material. If material is not included in the article's Creative Commons licence and your intended use is not permitted by statutory regulation or exceeds the 
permitted use, you will need to obtain permission directly from the copyright holder. To view a copy of this licence, visit http:// creativecommons.org/licenses/by-nc/4.0/.

\section{REFERENCES}

1. Bassetti M, Righi E, Carnelutti A, Graziano E, Russo A. Multidrug-resistant Klebsiella pneumoniae: challenges for treatment, prevention and infection control. Expert Rev Anti Infect Ther. 2018;16(10): 749-61. https://doi.org/10.1080/14787210.2018. 1522249 .

2. Martin RM, Bachman MA. Colonization, infection, and the accessory genome of Klebsiella pneumoniae. Front Cell Infect Microbiol. 2018;8:4.

3. van Duin D, Paterson DL. Multidrug-resistant bacteria in the community: an update. Infect Dis Clin North Am. 2020;34(3):709-22.

4. Richelsen R, Smit J, Schønheyder HC, et al. Outcome of community-onset ESBL-producing Escherichia coli and Klebsiella pneumoniae bacteraemia and urinary tract infection: a population-based cohort study in Denmark. J Antimicrob Chemother. 2020;75(12):3656-64.

5. Richelsen R, Smit J, Anru PL, Schønheyder HC, Nielsen $\mathrm{H}$. Incidence of community-onset extended-spectrum $\beta$-lactamase-producing Escherichia coli and Klebsiella pneumoniae infections: an 11-year population-based study in Denmark. Infect Dis (Lond). 2020;52(8):547-56.

6. Parrott AM, Shi J, Aaron J, Green DA, Whittier S, Wu F. Detection of multiple hypervirulent Klebsiella pneumoniae strains in a New York City hospital through screening of virulence genes. Clin Microbiol Infect. 2020;27(4):583-9.

7. Huynh BT, Passet V, Rakotondrasoa A, et al. Klebsiella pneumoniae carriage in low-income countries: antimicrobial resistance, genomic diversity and risk factors. Gut Microbes. 2020;11(5):1287-99.

8. Russo TA, Marr CM. Hypervirulent Klebsiella pneumoniae. Clin Microbiol Rev. 2019;32(3): e00001-19. https://doi.org/10.1128/CMR.-19 (Print 2019 Jun 19).

9. Ballot DE, Bandini R, Nana T, et al. A review of multidrug-resistant Enterobacteriaceae in a neonatal unit in Johannesburg, South Africa. BMC Pediatr. 2019;19(1):320.
10. Justo-da-Silva LH, De-Azeredo AN, Bueno AC, et al. Diversity of clonal types of Klebsiella pneumoniae causing infections in intensive care neonatal patients in a large urban setting. Braz J Microbiol. 2019;50(4):935-42.

11. Sankar MJ, Agarwal R, Deorari AK, Paul VK. Sepsis in the newborn. Indian J Pediatr. 2008;75(3):261-6.

12. Sands K, Carvalho MJ, Portal E, et al. Characterization of antimicrobial-resistant Gram-negative bacteria that cause neonatal sepsis in seven low- and middle-income countries. Nat Microbiol. 2021;6(4): 512-23.

13. Ernst CM, Braxton JR, Rodriguez-Osorio CA, et al. Adaptive evolution of virulence and persistence in carbapenem-resistant Klebsiella pneumoniae. Nat Med. 2020;26(5):705-11.

14. Caneiras C, Lito L, Melo-Cristino J, Duarte A. Community- and hospital-acquired Klebsiella pneumoniae urinary tract infections in Portugal: virulence and antibiotic resistance. Microorganisms. 2019;7(5):138.

15. Cilloniz C, Dominedo C, Torres A. Multidrug resistant gram-negative bacteria in community-acquired pneumonia. Crit Care. 2019;23(1):79.

16. Li B, Zhao Y, Liu C, Chen Z, Zhou D. Molecular pathogenesis of Klebsiella pneumoniae. Future Microbiol. 2014;9(9):1071-81.

17. Reza A, Sutton JM, Rahman KM. Effectiveness of efflux pump inhibitors as biofilm disruptors and resistance breakers in gram-negative (ESKAPEE) bacteria. Antibiotics (Basel). 2019;8(4):229.

18. Wyres KL, Lam MMC, Holt KE. Population genomics of Klebsiella pneumoniae. Nat Rev Microbiol. 2020;18(6):344-59. https://doi.org/10.1038/ s41579-019-0315-1.

19. Wyres KL, Holt KE. Klebsiella pneumoniae as a key trafficker of drug resistance genes from environmental to clinically important bacteria. Curr Opin Microbiol. 2018;45:131-9.

20. Pitout JD, Nordmann P, Poirel L. Carbapenemaseproducing Klebsiella pneumoniae, a key pathogen set for global nosocomial dominance. Antimicrob Agents Chemother. 2015;59(10):5873-84.

21. Zhong H, Chen F, Li YJ, et al. Global trends and hotspots in research of carbapenem-resistant Enterobacteriaceae (CRE): a bibliometric analysis from 2010 to 2020. Ann Palliat Med. 2021;10(6): 6079-91.

22. World Health Organization. Antimicrobial resistance: global report on surveillance. 2014. https:// 
apps.who.int/iris/bitstream/handle/10665/112642/ 9789241564748_eng.pdf;jsessionid=

E5C8037DF849A79FE72BF1

A6E3CCB14C?sequence=1. Accessed 16 June 2021.

23. Shields RK, Chen L, Cheng S, et al. Emergence of ceftazidime-avibactam resistance due to plasmidborne blaKPC-3 mutations during treatment of carbapenem-resistant Klebsiella pneumoniae infections. Antimicrob Agents Chemother. 2017. https:// doi.org/10.1128/AAC.02097-16.

24. van Duin D, Bonomo RA. Ceftazidime/avibactam and ceftolozane/tazobactam: second-generation $\beta$ lactam/ $\beta$-lactamase inhibitor combinations. Clin Infect Dis. 2016;63(2):234-41.

25. Hsieh PF, Lin TL, Lee CZ, Tsai SF, Wang JT. Seruminduced iron-acquisition systems and TonB contribute to virulence in Klebsiella pneumoniae causing primary pyogenic liver abscess. J Infect Dis. 2008;197(12):1717-27.

26. Holden VI, Breen P, Houle S, Dozois CM, Bachman MA. Klebsiella pneumoniae siderophores induce inflammation, bacterial dissemination, and HIF- $1 \alpha$ stabilization during pneumonia. MBio. 2016. https://doi.org/10.1128/mBio.01397-16.

27. Cooper JM, Rowley D. Resistance to Klebsiella pneumoniae and the importance of two bacterial antigens. Aust J Exp Biol Med Sci. 1982;60(6): $629-41$.

28. Mueller HL, Lanz M. Hyposensitization with bacterial vaccine in infectious asthma. A double-blind study and a longitudinal study. JAMA. 1969;208(8): 1379-83.

29. Rytel MW, Ferstenfeld JE, Rose HD, Balay J, Pierce WE, Lynch KL. Efficacy of a "mixed bacterial vaccine" in prophylaxis of acute respiratory infections: possible role of interferon. Am J Epidemiol. 1974;99(5):347-59.

30. Béné MC, Kahl L, Perruchet AM, et al. Bacterial lysates and ribosomes as inducers of specific immune responses: a comparative study. Scand J Immunol. 1993;38(5):496-8.

31. Lauener PA. Bacterial lysates and ribosomes as inducers of specific immune responses: a comparative study. Scand J Immunol. 1994;40(4):466-7.

32. Petrunov B, Marinova S, Markova R, et al. Cellular and humoral systemic and mucosal immune responses stimulated in volunteers by an oral polybacterial immunomodulator "Dentavax." Int Immunopharmacol. 2006;6(7):1181-93.

33. Nikolova M, Stankulova D, Taskov H, Nenkov P, Maximov V, Petrunov B. Polybacterial immunomodulator Respivax restores the inductive function of innate immunity in patients with recurrent respiratory infections. Int Immunopharmacol. 2009;9(4):425-32.

34. Yang B, Foley S. First experience in the UK of treating women with recurrent urinary tract infections with the bacterial vaccine Uromune( $($ ) $)$. BJU Int. 2018;121(2):289-92. https://doi.org/10.1111/ bju. 14067 .

35. Ramírez Sevilla C, Gómez Lanza E, Manzanera JL, Martín JAR, Sanz MÁB. Active immunoprophyilaxis with Uromune ${ }^{\circledR}$ decreases the recurrence of urinary tract infections at three and six months after treatment without relevant secondary effects. BMC Infect Dis. 2019;19(1):901.

36. Uehling DT, Hopkins WJ, Beierle LM, Kryger JV, Heisey DM. Vaginal mucosal immunization for recurrent urinary tract infection: extended phase II clinical trial. J Infect Dis. 2001;183(Suppl 1):S81-3.

37. Uehling DT, Hopkins WJ, Elkahwaji JE, Schmidt DM, Leverson GE. Phase 2 clinical trial of a vaginal mucosal vaccine for urinary tract infections. J Urol. 2003;170(3):867-9.

38. Hopkins WJ, Elkahwaji J, Beierle LM, Leverson GE, Uehling DT. Vaginal mucosal vaccine for recurrent urinary tract infections in women: results of a phase 2 clinical trial. J Urol. 2007;177(4):1349-53 (quiz 591).

39. Nenkov P. Overview on the clinical studies with Urostim immunostimulator against urogenital infections. Adv Exp Med Biol. 2000;485:325-9.

40. Rossi GA, Peri C, Raynal ME, et al. Naturally occurring immune response against bacteria commonly involved in upper respiratory tract infections: analysis of the antigen-specific salivary $\operatorname{IgA}$ levels. Immunol Lett. 2003;86(1):85-91.

41. Tricarico D, Varricchio A, D’Ambrosio S, Ascione E, Motta G. Prevention of recurrent upper respiratory tract infections in a community of cloistered nuns using a new immunostimulating bacterial lysate. A randomized, double-blind clinical trial. Arzneimittelforschung. 2004;54(1):57-63.

42. Macchi A, Vecchia LD. Open comparative, randomized controlled clinical study of a new immunostimulating bacterial lysate in the prophylaxis of upper respiratory tract infections. Arzneimittelforschung. 2005;55(5):276-81.

43. Cazzola M, Noschese P, Di Perna F. Value of adding a polyvalent mechanical bacterial lysate to therapy of COPD patients under regular treatment with salmeterol/fluticasone. Ther Adv Respir Dis. 2009;3(2):59-63. 
44. Braido F, Melioli G, Cazzola M, et al. Sub-lingual administration of a polyvalent mechanical bacterial lysate (PMBL) in patients with moderate, severe, or very severe chronic obstructive pulmonary disease (COPD) according to the GOLD spirometric classification: a multicentre, double-blind, randomised, controlled, phase IV study (AIACE study: advanced immunological approach in COPD exacerbation). Pulm Pharmacol Ther. 2015;33:75-80.

45. Braido F, Melioli G, Candoli P, et al. The bacterial lysate Lantigen $B$ reduces the number of acute episodes in patients with recurrent infections of the respiratory tract: the results of a double blind, placebo controlled, multicenter clinical trial. Immunol Lett. 2014;162(2 Pt B):185-93.

46. Mora R, Salzano FA, Mora E, Guastini L. Efficacy of a topical suspension of bacterial antigens for the management of chronic suppurative otitis media. Eur Arch Otorhinolaryngol. 2012;269(6):1593-7.

47. Mora R, Bellussi L, Passali FM, et al. Efficacy of a topical suspension of bacterial antigens for the management of recurrent eczema in children. Med Sci Monit. 2004;10(9):Pi99-pi103.

48. Opoku-Temeng C, Kobayashi SD, DeLeo FR. Klebsiella pneumoniae capsule polysaccharide as a target for therapeutics and vaccines. Comput Struct Biotechnol J. 2019;17:1360-6.

49. Choi M, Tennant SM, Simon R, Cross AS. Progress towards the development of Klebsiella vaccines. Expert Rev Vaccines. 2019;18(7):681-91. https:// doi.org/10.1080/14760584.2019.1635460.

50. Micoli F, Costantino P, Adamo R. Potential targets for next generation antimicrobial glycoconjugate vaccines. FEMS Microbiol Rev. 2018;42(3):388-423.

51. Patro LPP, Rathinavelan T. Targeting the sugary armor of Klebsiella species. Front Cell Infect Microbiol. 2019;9:367.

52. Pletz MW, Uebele J, Gotz K, Hagel S, BekeredjianDing I. Vaccines against major ICU pathogens: where do we stand? Curr Opin Crit Care. 2016;22(5):470-6.

53. Choi M, Hegerle N, Nkeze J, et al. The diversity of lipopolysaccharide $(\mathrm{O})$ and capsular polysaccharide (K) antigens of invasive Klebsiella pneumoniae in a multi-country collection. Front Microbiol. 2020;11: 1249.

54. Follador R, Heinz E, Wyres KL, et al. The diversity of Klebsiella pneumoniae surface polysaccharides. Microb Genom. 2016;2(8): e000073.

55. Feldman MF, Mayer Bridwell AE, Scott NE, et al. A promising bioconjugate vaccine against hypervirulent Klebsiella pneumoniae. Proc Natl Acad Sci USA. 2019;116(37):18655-63.

56. Cryz SJ Jr, Fürer E, Germanier R. Safety and immunogenicity of Klebsiella pneumoniae K1 capsular polysaccharide vaccine in humans. J Infect Dis. 1985;151(4):665-71.

57. Edelman R, Taylor DN, Wasserman SS, et al. Phase 1 trial of a 24-valent Klebsiella capsular polysaccharide vaccine and an eight-valent Pseudomonas O-polysaccharide conjugate vaccine administered simultaneously. Vaccine. 1994;12(14):1288-94.

58. Campbell WN, Hendrix E, Cryz S Jr, Cross AS. Immunogenicity of a 24-valent Klebsiella capsular polysaccharide vaccine and an eight-valent Pseudomonas O-polysaccharide conjugate vaccine administered to victims of acute trauma. Clin Infect Dis. 1996;23(1):179-81.

59. Ravinder M, Liao KS, Cheng YY, et al. A synthetic carbohydrate-protein conjugate vaccine candidate against Klebsiella pneumoniae serotype K2. J Org Chem. 2020;85(24):15964-97.

60. Malachowa N, Kobayashi SD, Porter AR, et al. Vaccine protection against multidrug-resistant Klebsiella pneumoniae in a nonhuman primate model of severe lower respiratory tract infection. MBio. 2019. https://doi.org/10.1128/mBio.02994-19.

61. Paton JC, Trappetti C. Streptococcus pneumoniae capsular polysaccharide. Microbiol Spectr. 2019. https://doi.org/10.1128/microbiolspec.GPP3-00192018.

62. Briles DE, Paton JC, Mukerji R, Swiatlo E, Crain MJ. Pneumococcal vaccines. Microbiol Spectr. 2019. https://doi.org/10.1128/microbiolspec.GPP3-00282018.

63. Clarke BR, Ovchinnikova OG, Sweeney RP, et al. A bifunctional $\mathrm{O}$-antigen polymerase structure reveals a new glycosyltransferase family. Nat Chem Biol. 2020;16(4):450-7.

64. Cross AS, Opal SM, Warren HS, et al. Active immunization with a detoxified Escherichia coli J5 lipopolysaccharide group B meningococcal outer membrane protein complex vaccine protects animals from experimental sepsis. J Infect Dis. 2001;183(7):1079-86.

65. Bennett-Guerrero E, McIntosh TJ, Barclay GR, et al. Preparation and preclinical evaluation of a novel liposomal complete-core lipopolysaccharide vaccine. Infect Immun. 2000;68(11):6202-8.

66. Salles MF, Mandine E, Zalisz R, Guenounou M, Smets P. Protective effects of murine monoclonal antibodies in experimental septicemia: E. coli 
antibodies protect against different serotypes of E. coli. J Infect Dis. 1989;159(4):641-7.

67. Clements A, Jenney AW, Farn JL, et al. Targeting subcapsular antigens for prevention of Klebsiella pneumoniae infections. Vaccine. 2008;26(44): 5649-53.

68. Hsieh PF, Wu MC, Yang FL, et al. D-galactan II is an immunodominant antigen in $\mathrm{O} 1$ lipopolysaccharide and affects virulence in Klebsiella pneumoniae: implication in vaccine design. Front Microbiol. 2014;5:608.

69. Pennini ME, De Marco A, Pelletier M, et al. Immune stealth-driven $\mathrm{O} 2$ serotype prevalence and potential for therapeutic antibodies against multidrug resistant Klebsiella pneumoniae. Nat Commun. 2017;8(1): 1991.

70. Mandine E, Salles MF, Zalisz R, Guenounou M, Smets P. Murine monoclonal antibodies to Klebsiella pneumoniae protect against lethal endotoxemia and experimental infection with capsulated K. pneumoniae. Infect Immun. 1990;58(9):2828-33.

71. Chathley UC, Sharma S, Chhibber S. Lipopolysaccharide-induced resistance in mice against ascending urinary tract infection with Klebsiella pneumoniae. Folia Microbiol (Praha). 1996;41(4): 373-6.

72. Chhibber S, Bajaj J. Polysaccharide-iron-regulated cell surface protein conjugate vaccine: its role in protection against Klebsiella pneumoniae-induced lobar pneumonia. Vaccine. 1995;13(2):179-84.

73. Hegerle N, Choi M, Sinclair J, et al. Development of a broad spectrum glycoconjugate vaccine to prevent wound and disseminated infections with Klebsiella pneumoniae and Pseudomonas aeruginosa. PLoS ONE. 2018;13(9): e0203143.

74. Cross TBA, Simon R, Michon F, et al. Novel multivalent vaccine for gram-negative bacterial pathogens, including multiple antibiotic-resistant strains. World Vaccine Congress, April 14-17, 2019. Washington DC.

75. Lee JC, Lee EJ, Lee JH, et al. Klebsiella pneumoniae secretes outer membrane vesicles that induce the innate immune response. FEMS Microbiol Lett. 2012;331(1):17-24.

76. Martora F, Pinto F, Folliero V, et al. Isolation, characterization and analysis of pro-inflammatory potential of Klebsiella pneumoniae outer membrane vesicles. Microb Pathog. 2019;136: 103719.

77. Jan AT. Outer membrane vesicles (OMVs) of gramnegative bacteria: a perspective update. Front Microbiol. 2017;8:1053.
78. Turner KL, Cahill BK, Dilello SK, et al. Porin loss impacts the host inflammatory response to outer membrane vesicles of Klebsiella pneumoniae. Antimicrob Agents Chemother. 2015;60(3):1360-9.

79. Lee WH, Choi HI, Hong SW, Kim KS, Gho YS, Jeon SG. Vaccination with Klebsiella pneumoniae-derived extracellular vesicles protects against bacteria-induced lethality via both humoral and cellular immunity. Exp Mol Med. 2015;47(9): e183. https:// doi.org/10.1038/emm.2015.59.

80. Wu G, Ji H, Guo X, et al. Nanoparticle reinforced bacterial outer-membrane vesicles effectively prevent fatal infection of carbapenem-resistant Klebsiella pneumoniae. Nanomedicine. 2020;24: 102148.

81. Tan K, Li R, Huang X, Liu Q. Outer membrane vesicles: current status and future direction of these novel vaccine adjuvants. Front Microbiol. 2018;9: 783.

82. Gregory RL. Microbial ribosomal vaccines. Rev Infect Dis. 1986;8(2):208-17.

83. Robert D, Michel S, Ivanoff B, Cozzone AJ, Fontanges R. On the immunogenicity of ribosomes and ribosomal proteins isolated from Klebsiella pneumoniae and Streptococcus pneumoniae. Microbiol Immunol. 1981;25(2):183-94.

84. Robert D, Ivanoff B, Fontanges R, Normier G, Pinel AM, Dussourd DL. Study of the mode of action of ribosomal vaccines from Klebsiella and Streptococcus pneumoniae and their ribonucleic and protein fractions using passive immunization. Microbiol Immunol. 1982;26(10):933-40.

85. Michel FB, Dussourd D'Hinterland L, Bousquet J, Pinel AM, Normier G. Immuno-stimulation by a ribosomal vaccine associated with a bacterial cell wall adjuvant in humans. Infect Immun. 1978;20(3):760-9.

86. Serrano E, Demanez JP, Morgon A, Chastang C, Van Cauwenberge P. Effectiveness of ribosomal fractions of Klebsiella pneumoniae, Streptococcus pneumoniae, Streptococcus pyogenes, Haemophilus influenzae and the membrane fraction of Kp (Ribomunyl) in the prevention of clinical recurrences of infectious rhinitis. Results of a multicenter double-blind placebo-controlled study. Eur Arch Otorhinolaryngol. $1997 ; 254(8): 372-5$.

87. Pregliasco F, Terracciano L, Marcassa S, Zava D, Anselmi G. Rationale for the clinical use of a ribosome-component immune modulator. Allergy Asthma Proc. 2009;30(Suppl 1):S5-12.

88. Haeuw JF, Rauly I, Zanna L, et al. The recombinant Klebsiella pneumoniae outer membrane protein 
OmpA has carrier properties for conjugated antigenic peptides. Eur J Biochem. 1998;255(2):446-54.

89. Goetsch L, Gonzalez A, Plotnicky-Gilquin H, et al. Targeting of nasal mucosa-associated antigen-presenting cells in vivo with an outer membrane protein A derived from Klebsiella pneumoniae. Infect Immun. 2001;69(10):6434-44.

90. Soulas C, Baussant T, Aubry JP, et al. Outer membrane protein A (OmpA) binds to and activates human macrophages. J Immunol. 2000;165(5): 2335-40.

91. Rauly I, Goetsch L, Haeuw JF, et al. Carrier properties of a protein derived from outer membrane protein A of Klebsiella pneumoniae. Infect Immun. 1999;67(11):5547-51.

92. Babu L, Uppalapati SR, Sripathy MH, Reddy PN. Evaluation of recombinant multi-epitope outer membrane protein-based Klebsiella pneumoniae subunit vaccine in mouse model. Front Microbiol. 2017;8:1805.

93. Hussein KE, Bahey-El-Din M, Sheweita SA. Immunization with the outer membrane proteins OmpK17 and OmpK36 elicits protection against Klebsiella pneumoniae in the murine infection model. Microb Pathog. 2018;119:12-8.

94. Kurupati P, Ramachandran NP, Poh CL. Protective efficacy of DNA vaccines encoding outer membrane protein A and OmpK36 of Klebsiella pneumoniae in mice. Clin Vaccine Immunol. 2011;18(1):82-8. https://doi.org/10.1128/CVI.00275-10.

95. Serushago BA, Mitsuyama M, Handa T, Koga T, Nomoto K. Role of antibodies against outer-membrane proteins in murine resistance to infection with encapsulated Klebsiella pneumoniae. J Gen Microbiol. 1989;135(8):2259-68.

96. Talukdar S, Bayan U, Saikia KK. In silico identification of vaccine candidates against Klebsiella oxytoca. Comput Biol Chem. 2017;69:48-54.

97. Dar HA, Zaheer T, Shehroz M, et al. Immunoinformatics-aided design and evaluation of a potential multi-epitope vaccine against Klebsiella pneumoniae. Vaccines (Basel). 2019;7(3):88.

98. Mehmood A, Naseer S, Ali A, Fatimah H, Rehman S, Kiani A. Identification of novel vaccine candidates against carbapenem resistant Klebsiella pneumoniae: a systematic reverse proteomic approach. Comput Biol Chem. 2020;89: 107380.

99. Lienard D, Avril MF, Le Gal FA, et al. Vaccination of melanoma patients with Melan-A/Mart-1 peptide and Klebsiella outer membrane protein p40 as an adjuvant. J Immunother. 2009;32(8):875-83.
100. Libon C, Haeuw JF, Crouzet F, et al. Streptococcus pneumoniae polysaccharides conjugated to the outer membrane protein A from Klebsiella pneumoniae elicit protective antibodies. Vaccine. 2002;20(17-18):2174-80.

101. Hornick DB, Thommandru J, Smits W, Clegg S. Adherence properties of an mrkD-negative mutant of Klebsiella pneumoniae. Infect Immun. 1995;63(5): 2026-32.

102. Jagnow J, Clegg S. Klebsiella pneumoniae MrkD-mediated biofilm formation on extracellular matrixand collagen-coated surfaces. Microbiology. 2003;149(Pt 9):2397-405. https://doi.org/10.1099/ mic.0.26434-0.

103. Gomes AÉI, Pacheco T, Dos Santos CDS, et al. Functional insights from KpfR, a new transcriptional regulator of fimbrial expression that is crucial for Klebsiella pneumoniae pathogenicity. Front Microbiol. 2020;11: 601921.

104. Witkowska D, Mieszała M, Gamian A, et al. Major structural proteins of type 1 and type 3 Klebsiella fimbriae are effective protein carriers and immunogens in conjugates as revealed from their immunochemical characterization. FEMS Immunol Med Microbiol. 2005;45(2):221-30. https://doi.org/ 10.1016/j.femsim.2005.04.005.

105. Li Y, Li ZJ, Han WY, et al. Identification and characterization of Th cell epitopes in MrkD adhesin of Klebsiella pneumoniae. Microb Pathog. 2010;49(1-2): 8-13. https://doi.org/10.1016/j.micpath.2010.03. 009.

106. Wang Q, Chang CS, Pennini M, et al. Target-agnostic identification of functional monoclonal antibodies against Klebsiella pneumoniae multimeric MrkA fimbrial subunit. J Infect Dis. 2016;213(11): 1800-8. https://doi.org/10.1093/infdis/jiw021.

107. Wang Q, Chen Y, Cvitkovic R, et al. Anti-MrkA monoclonal antibodies reveal distinct structural and antigenic features of MrkA. PLoS ONE. 2017;12(1):e0170529. https://doi.org/10.1371/ journal.pone.0170529.

108. Lavender H, Jagnow JJ, Clegg S. Klebsiella pneumoniae type 3 fimbria-mediated immunity to infection in the murine model of respiratory disease. Int $\mathrm{J}$ Med Microbiol. 2005;295(3):153-9. https://doi.org/ 10.1016/j.ijmm.2005.04.001.

109. Langermann S, Möllby R, Burlein JE, et al. Vaccination with FimH adhesin protects cynomolgus monkeys from colonization and infection by uropathogenic Escherichia coli. J Infect Dis. 2000;181(2): 774-8. https://doi.org/10.1086/315258. 
110. Zandi M, Fallah Mehrabadi J, Mahdavi M, Irani S. Construction and development of FimH lectin domain for rising immune response after injection by uropathogenic E.coli. Hum Antibodies. 2020;28(2):169-78. https://doi.org/10.3233/HAB200404.

111. Thankavel $\mathrm{K}$, Madison B, Ikeda $\mathrm{T}$, et al. Localization of a domain in the FimH adhesin of Escherichia coli type 1 fimbriae capable of receptor recognition and use of a domain-specific antibody to confer protection against experimental urinary tract infection. J Clin Invest. 1997;100(5):1123-36. https://doi.org/ 10.1172/JCI119623.

112. Poggio TV, La Torre JL, Scodeller EA. Intranasal immunization with a recombinant truncated $\mathrm{FimH}$ adhesin adjuvanted with CpG oligodeoxynucleotides protects mice against uropathogenic Escherichia coli challenge. Can J Microbiol. 2006;52(11):1093-102. https://doi.org/10.1139/ w06-065.

113. Asadi Karam MR, Habibi M, Bouzari S. Use of flagellin and cholera toxin as adjuvants in intranasal vaccination of mice to enhance protective immune responses against uropathogenic Escherichia coli antigens. Biologicals. 2016;44(5):378-86. https:// doi.org/10.1016/j.biologicals.2016.06.006.

114. Asadi Karam MR, Oloomi M, Mahdavi M, Habibi M, Bouzari S. Vaccination with recombinant FimH fused with flagellin enhances cellular and humoral immunity against urinary tract infection in mice. Vaccine. 2013;31(8):1210-6. https://doi.org/10. 1016/j.vaccine.2012.12.059.

115. Asadi KM, Oloomi M, Habibi M, Bouzari S. Cloning of fimH and fliC and expression of the fusion protein FimH/FliC from Uropathogenic Escherichia coli (UPEC) isolated in Iran. Iran J Microbiol. 2012;4(2): $55-62$.

116. Karam MR, Oloomi M, Mahdavi M, Habibi M, Bouzari S. Assessment of immune responses of the flagellin (FliC) fused to FimH adhesin of uropathogenic Escherichia coli. Mol Immunol. 2013;54(1): 32-9. https://doi.org/10.1016/j.molimm.2012.11. 002.

117. Savar NS, Jahanian-Najafabadi A, Mahdavi M, Shokrgozar MA, Jafari A, Bouzari S. In silico and in vivo studies of truncated forms of flagellin (FliC) of enteroaggregative Escherichia coli fused to FimH from uropathogenic Escherichia coli as a vaccine candidate against urinary tract infections. J Biotechnol. 2014;175:31-7.

118. Fan X, Yue Y, Xiong S. Incorporation of a bi-functional protein FimH enhances the immunoprotection of chitosan-pVP1 vaccine against coxsackievirus B3-induced myocarditis. Antiviral Res. 2017;140:121-32.

119. Fernández-García L, Blasco L, Lopez M, et al. Toxinantitoxin systems in clinical pathogens. Toxins (Basel). 2016;8(7):227.

120. Choby JE, Howard-Anderson J, Weiss DS. Hypervirulent Klebsiella pneumonia-clinical and molecular perspectives. J Intern Med. 2020;287(3):283-300.

121. Faïs T, Delmas J, Barnich N, Bonnet R, Dalmasso G. Colibactin: more than a new bacterial toxin. Toxins (Basel). 2018;10(4):151.

122. Klipstein FA, Engert RF, Houghten RA. Immunological properties of purified Klebsiella pneumoniae heat-stable enterotoxin. Infect Immun. 1983;42(2): 838-41.

123. Singh BR, Sharma VD. Potential of Klebsiella pneumoniae cytotoxin toxoid as vaccine against klebsiellosis in rabbits and mice. Vaccine. 2001;19(31):4505-10. https://doi.org/10.1016/ s0264-410x(01)00187-6.

124. Gorden PJ, Kleinhenz MD, Ydstie JA, et al. Efficacy of vaccination with a Klebsiella pneumoniae siderophore receptor protein vaccine for reduction of Klebsiella mastitis in lactating cattle. J Dairy Sci. 2018;101(11):10398-408. https://doi.org/10.3168/ jds.2017-4267.

125. Kumar A, Harjai K, Chhibber S. Early cytokine response to lethal challenge of Klebsiella pneumoniae averted the prognosis of pneumonia in FyuA immunized mice. Microb Pathog. 2020;144: 104161.

126. Lundberg U, Senn BM, Schüler W, Meinke A, Hanner $M$. Identification and characterization of antigens as vaccine candidates against Klebsiella pneumoniae. Hum Vaccin Immunother. 2013;9(3): 497-505. https://doi.org/10.4161/hv.23225.

127. Kurupati P, Teh BK, Kumarasinghe G, Poh CL. Identification of vaccine candidate antigens of an ESBL producing Klebsiella pneumoniae clinical strain by immunoproteome analysis. Proteomics. 2006;6(3):836-44.

128. Rodrigues MX, Yang Y, de Souzameira EB Jr, do Carmo Silva SJ, Bicalho RC. Development and evaluation of a new recombinant protein vaccine (YidR) against Klebsiella pneumoniae infection. Vaccine. 2020;38(29):4640-8.

129. Tomazi T, Tomazi A, Silva JCC, et al. Immunization with a novel recombinant protein (YidR) reduced the risk of clinical mastitis caused by Klebsiella spp. and decreased milk losses and culling risk after 
Escherichia coli infections. J Dairy Sci. 2021;104(4): 4787-802.

130. Lin J, Hogan JS, Smith KL. Antigenic homology of the inducible ferric citrate receptor (FecA) of coliform bacteria isolated from herds with naturally occurring bovine intramammary infections. Clin Diagn Lab Immunol. 1999;6(6):966-9.

131. Takemura K, Hogan JS, Smith KL. Effect of immunoglobulin $G$ from cows immunized with ferric citrate receptor (FecA) on iron uptake by Escherichia coli. J Dairy Sci. 2003;86(1):133-7.

132. Wolf SL, Hogan JS, Smith KL. Iron uptake by Escherichia coli cultured with antibodies from cows immunized with high-affinity ferric receptors. J Dairy Sci. 2004;87(7):2103-7.
133. Takemura K, Hogan JS, Lin J, Smith KL. Efficacy of immunization with ferric citrate receptor FecA from Escherichia coli on induced coliform mastitis. J Dairy Sci. 2002;85(4):774-81.

134. Converso TR, Assoni L, Andre GO, Darrieux M, Leite LCC. The long search for a serotype independent pneumococcal vaccine. Expert Rev Vaccines. 2020;19(1):57-70.

\section{Publisher's Note}

Springer Nature remains neutral with regard to jurisdictional claims in published maps and institutional affiliations. 